\title{
Mechanical, thermal and electromagnetic properties of nanographite platelets modified cementitious composites
}

\author{
Xia Cui ${ }^{1}$, Shengwei Sun ${ }^{2}$, Baoguo Han ${ }^{1, *}$, Xun $\mathrm{Yu}^{3,4}$, Jian Ouyang ${ }^{5}$, Shuzhu Zeng ${ }^{1}$, Jinping Ou ${ }^{1,2}$ \\ ${ }^{1}$ School of Civil Engineering, Dalian University of Technology, Dalian 116024, China \\ ${ }^{2}$ School of Civil Engineering, Harbin Institute of Technology, Harbin 150090, China \\ ${ }^{3}$ Department of Mechanical Engineering, New York Institute of Technology, New York 11568, USA \\ ${ }^{4}$ School of Machinery and Automation, Wuhan University of Science and Technology, Wuhan, 430081 China \\ ${ }^{5}$ School of Transportation and Logistics, Dalian University of Technology, Dalian, 116024 China \\ *Corresponding author: hithanbaoguo@163.com, hanbaoguo@dlut.edu.cn
}

\section{Abstract}

Nanographite platelets (NGPs) are used as multi-functional fillers to develop cementitious composites

because the unique carbon-carbon hexagonal plane structure makes NGPs have excellent mechanical,

electrical, dielectric and thermal properties. In this paper, cementitious composites filled with NGPs are

fabricated to investigate the effect of NGPs on mechanical, thermal and electromagnetic properties of

cementitious composites. Experiment results show that the addition of NGPs can effectively modify the above-mentioned properties of cementitious composites. When the content of NGPs reaches 5\%, the hardness of the composites increases 1.5 -fold, the abrasive loss per unit area of the composites reduces by $71 \%$, the abrasion depth of the composites reduces by $73 \%$, the thermal conductivity of the composites increases by $77 \%$, the specific heat of the composites decreases by $17.7 \%$, the damping ratio of the composites increases by $20 \%$, and the electromagnetic wave reflectivity of the composites decreases by $38 \%$ compared with pure cementitious composites.

Keywords: A. Nanocomposites; A. Graphene; B. Mechanical properties; B. Thermal properties 


\section{Introduction}

Cementitious composite is one of the most widely used construction materials in the world because they have abundant resources, strong adaptability and mature production process. However, with the development of modern concrete structure with large-scale and intelligent trend, single function of traditional cementitious composite has not been able to meet the requirement of multifunctional infrastructures. Modern infrastructures have posed new challenges for cementitious materials, not only requiring to high bearing loading capability, but also possessing the specific or multiple functions in various aspects such as electricity, heat, sound, light and magnetism. This also provides a good opportunity for the research and development of new functional cementitious composites [1-3]. Nanotechnology has changed and will continue to change our vision, expectations and abilities to control the material world [4]. These developments will definitely affect the field of construction and construction materials. Nano nonmetallic oxide and metallic oxide similar to cementitious composites are first used to enhance/modify cementitious materials. Moreover, extensive research endeavors demonstrated the potential of various nano carbon materials including carbon nanotubes, carbon nanofibres, and nano carbon black for enhancing/modifying cementitious materials [5-10]. However, at present the research on nanographite platelets (NGPs) modified cementitious composites just starts [1, 11-15].

NGPs are a new type of functional fillers for cementitious composite which consist of small stacks of graphene, and are usually $0.35-100 \mathrm{~nm}$ thick. As we know, graphene is a single layer carbon-carbon hexagonal plane named as "sub structure unit". Therefore, NGPs are composed of multilayer net-like structure of carbon-carbon hexagonal plane. In return, graphene is regarded as a special case of NGPs which has only one carbon atoms layer. Consequently, it is believed that the properties of NGPs are close 
1 to graphene [16-18]. In recent years, the nanocomposites represented by NGPs have been paid to more

2 and more attention in various fields [19-21]. The existing studies have showed that the hardness of single

3 layer graphite sheet is more than $100 \mathrm{GPa}$ and harder than diamond [22]. Therefore, adding NGPs into the

4 cementitious composites has the potential to improve the hardness of the cementitious composites. The

5 improvement of the hardness will inevitably lead to the improvement of the abrasion resistance

6 performance of the cementitious composites. Moreover, the lubrication effect of NGPs can be also helpful

7 to improve the abrasion resistance performance of the cementitious composites. In fact, several

8 researchers have reported good abrasion resistance performance of NGPs filled composites [23-28]. Du et

9 al. prepared $\mathrm{Cu}$-based friction materials with nano-graphite through powder metallurgy technology. The

10 results indicated the nano-graphite particles could reduce the abrasion and enhance the abrasion resistance

11 performance of the $\mathrm{Cu}$-based friction materials. Compared with the friction materials without nano- $\mathrm{Cu}$,

12 the abrasion resistance and heat resistance of the friction materials with nano-graphite had been improved

13 by $11 \%$ and $25 \%$, respectively [26]. Liao's experimental research showed that multilayer graphite had

14 lower friction property of graphene than the single layer graphene [27]. Huang added the NGPs into

15 bismaleimide (IBM) to make a new abrasion resistance composite. Moreover, the abrasion resistance of

16 small amount of NGPs (3\%-5\%) could achieve the same effect with the addition of $20 \%$ graphite powder $17[28]$.

The aluminum-based composite filled with graphite particles was prepared by Cheng et al. The

19 damping property of the composite increased as the content of the graphite particles increased, and the

20 damping property of the composites was several times higher than that of graphite and pure aluminum

21 [29]. Liu et al. prepared n-Gr-Al-Mg-Si composites by using the hot pressing method and observed that the addition of nanographite could significantly improve the damping behavior of composite materials. 
1 The damping factor of Gr-Al-Mg composite increased by about $10 \%$ [30]. Huang et al. reported that damping property of the composites enhanced with the decreasing size of graphite particle under the same

volume fraction of macroscopic graphite particular, so small particle NGPs was helpful to improve the damping property of composite[31]. Muthusamy et al. prepared a cementitious graphite network composite containing 8 vol. \% graphite, which had unexpected vibration damping because this material had high values of both the loss tangent (vibration amplitude decay rate) and the loss modulus (energy dissipation ability, equal to the product of the storage modulus and the loss tangent) [32].

Faugeraset et al. investigated the thermal conductivity of graphene in Corbino Membrane Geometry and proved that graphene was a good conductor of heat [33]. Some researches have been done on thermal conductivity of NGPs/polymer composites. Chandrasekaran et al. reported that the increase percentage in thermal conductivity of the NGP/epoxy was $11 \%$ for $1.0 \mathrm{wt} \%$ and $14 \%$ for $2.0 \mathrm{wt} \%$ loading filler [34].

Raza et al. reported that NGP/silicone composite containing 20 wt.\% NGPs had a thermal conductivity of $1.909 \mathrm{~W} /(\mathrm{m} . \mathrm{K})$ which is an 11 -fold increase over that of pure silicone $(0.175 \mathrm{~W} / \mathrm{m} . \mathrm{K})$ [35]. Yu et al. reported that NGPs (no more than 4 graphene layer) provided a thermal conductivity of NGP/epoxy enhancement of more than $3000 \%$ (loading of $25 \mathrm{vol} . \%$ ), and a thermal conductivity reached $6.44 \mathrm{~W} / \mathrm{m} . \mathrm{K}$, which surpassed the performance of conventional fillers that required a loading of 70 vol.\% to achieve these values [36]. Sedaghat et al. observed that the addition of graphite could improve the thermal conductivity of cement and reduce the early hydration heat of cement [18].

The excellent conductive property and high dielectric loss angle of graphite sheets stimulates the scientific researchers to explore the electromagnetic wave absorbing property of the graphite/polymer composites. The study of Guo showed that the content of graphite had a certain influence on the electromagnetic wave absorbing property of cementitious materials [37]. A kind of cementitious 
composites capable of absorbing electromagnetic wave was made of graphite, expanded perlite and

2 Portland cement. The results showed that a proper amount of graphite was helpful to improve the

3 electromagnetic wave absorbing effect of cementitious composites. The composites had a good

4 electromagnetic wave absorbing performance in the $2-18 \mathrm{GHz}$ band when the content of graphite powder

5 was $20 \%$, and the minimum reflectivity could reach -26dB [38]. Han et al. showed that electromagnetic

6 wave absorbing performance of materials was linked to particle size of graphite. The small-size graphite

7 would be helpful for absorbing electromagnetic wave [39]. Shafieizadegan-Esfahani et al. developed the

8 polyurethane foam (PU) radiofrequency wave absorber with silicone rubber (SR) and graphite nanosheets

9 (GN). They found that all PU/SR/GN prepared nanocomposites exhibited electromagnetic wave reflection

10 loss behavior and this revealed to be affected by the GN level as well as the size and dispersion state of

11 the graphite nanosheets [40, 41]. Wang et al. reported that the polyvinylidene fluoride (PVDF)/graphite

12 intercalated composite (GIC) composites exhibited strong microwave absorbing intensities (64.4 dB at

$1310.59 \mathrm{GHz})$ with a low filler loading $(9.0 \mathrm{wt} \%)$ [42].

Based on the above analysis, it is clear that the modification of NGPs to performances of

15 polymer-matrix, metal-matrix and ceramic-matrix composites has been widely studied. Additionally,

16 some studies have investigated the reinforcement of NGPs to compressive and flexural properties,

17 durability, and strain sensing capability of cementitious composites. However, to authors' knowledge, few

researches have been done on hardness, abrasion resistance, damping, thermal conductivity, specific heat

19 and electromagnetic wave absorbing properties when NGPs are added into the cementitious composites.

20 Therefore, this paper will incorporate the NGPs into cementitious composites and study the effect of

21 NGPs on mechanical properties (including hardness, abrasion resistance and damping properties), thermal 
1 electromagnetic wave absorbing property) of cementitious composites.

2

3

4

5

6

7

8

\section{Experimental methods}

\subsection{Materials}

The raw materials for fabricating cementitious composites with and without NGPs are listed as following. P.O.42.5R Portland cement used as binder material is produced by Dalian Onoda Cement Company. NGPs were provided by Shanghai Ranen New Energy Equipment \& Technology Co.,Ltd., properties of which are shown in Table 1. The water reducing agent is polycarboxylate superplasticizer (Sika ViscoCrete 3301E), which is used to disperse NGPs and provided by Sika (China) Ltd., Dalian. Standard sand is used as the fine aggregate and purchased from Xiamen AI Ou Standard Sand Co.,Ltd.,China.

\subsection{Preparation of samples}

The mix proportions of the cementitious composites with NGPs are shown in Table 2. The experiment adopted the principle of a single variable that only the dosage of NGPs was changed. The water to cement ratio and the proportion of polycarboxylate superplasticizer remained the same in order to only study the effect of NGPs dosage on cementitious composites. The process of fabricating specimen is as follows.

Firstly, all the raw materials were weighed according to the mixing proportions. Secondly, water, polycarboxylate superplasticizer and NGPs were mixed together. An ultrasonicator (American Branson 2510 E-DTH provided by Bransonic Ultrasonics Corporation) is used to ultrasonic the suspension for $1 \mathrm{~h}$ with (100W 42KHz) for preparing uniform suspension. Thirdly, the cement was put slowly into the suspension of ultrasonic treatment and at the same time stirred with a Constant Speed Stirrer ( DW-2 DC provided by Chinese Yu Hua Instrument Ltd.) at low speed for 60s firstly, and then at high speed for 30s. Fourthly, the sand was put into mixing pot and at the same time stirred at low speed firstly for 60 s and 
1 then at high speed for 30s. Fifthly, the specimens after stirring were put into the corresponding oiled molds. There were totally four sizes of molds in the experiment. Mold used in wear test was standard cube of $150 \mathrm{~mm} \times 150 \mathrm{~mm} \times 150 \mathrm{~mm}$. The sizes of the samples for thermal conductivity, damping ratio and wave absorbing performance are $90 \mathrm{~mm} \times 90 \mathrm{~mm} \times 10 \mathrm{~mm}, 260 \mathrm{~mm} \times 20 \mathrm{~mm} \times 20 \mathrm{~mm}$ and $200 \mathrm{~mm} \times 200 \mathrm{~mm} \times 10 \mathrm{~mm}$, respectively. Then they were put onto the vibrating table until shaking to an extent of oozing slurry on the surface of specimens. Sixth, the specimens were demolded after $24 \mathrm{~h}$ and then cured the specimens for $28 \mathrm{~d}$ in the standard condition at temperature of $20^{\circ} \mathrm{C}$ and a relative humidity higher than $95 \%$. Finally, the specimens were dried at room temperature before testing. Three specimens in each group were used to test.

\subsection{Measurement}

\subsubsection{Test for hardness}

The Nano Indenter XP System (MTS Systems Corporation, America) is used to measure the hardness of cementitious composite. The specimens are loaded at a $4 \mathrm{~nm} / \mathrm{s}$ of loading rate until the penetration depth reaches $2 \mu \mathrm{m}$. The hardness value in the steady stage is taken as the nano hardness of the composites.

\subsubsection{Test for abrasion resistance}

Abrasion loss per unit area used to evaluate the abrasion resistance of cementitious composites was measured by an Abrasion Wear Test Machine (Model TMS-04, Wuxi Jianyi Instrument \& Machinery Co., Ltd. in China). After $27 \mathrm{~d}$ curing, the specimens were removed from the curing sites and seasoned for $12 \mathrm{~h}$ at room temperature, and then was baked in an oven at $60 \pm 5^{\circ} \mathrm{C}$ for $12 \mathrm{~h}$ to constant weight before testing.

20 The abrasion resistance test is as follows. The specimens were firstly milled about $30 \mathrm{rpm}$ at $200 \mathrm{~N}$ loading, and then the specimens were removed to weigh its mass after brushing off surface dust. The corresponding mass " $m_{l}$ " were recorded as the initial quality of the specimen. Next, the specimens were 
1 ground $60 \mathrm{rpm}$ at $200 \mathrm{~N}$ loading. Then the specimens were removed to weigh after brushing off surface

2 dust. At the same time, the residual mass of samples was recorded as " $m_{2}$ ". The abrasion amount of each

3 specimen is calculated through the following formula.

$$
G c=\left(m_{1}-m_{2}\right) / 0.0125
$$

4 where $G c$ was the abrasion loss per unit area, $m_{l}$ was the initial mass of specimen, and $m_{2}$ was residual mass of specimen being worn.

$6 \quad$ 2.3.3 Test for damping ratio

The time-domain exponential decay method is used to study the damping ratio of cementitious

member in this experiment. Damping ratio is a dimensionless quantity, which is an index on the capability of specimens in reducing vibration caused by excitation. The higher the value of damping ratio, the better the capability in reducing vibration caused by excitation.

Test instruments of time-domain exponential decay method mainly include an acceleration sensor

(PCB-353B33, PCB Piezotronics Inc.), a NI-9233(National Instruments Corporation, America) dynamic

Damping ratio of specimens is calculated according to the formula (2).

$$
\xi=\frac{1}{2 i \pi} \ln \left(\frac{a_{n}}{a_{n+i}}\right)
$$

where $\xi$ is the damping ratio, $a_{n}$ and $a_{n+i}$ are the acceleration amplitude of the peak value at " $i$ "-th and " $n+i$ "-th, respectively. damping ratio of three specimens in each group was regarded as the final results. 
The quasi-steady-state method, which requires constant temperature difference and temperature rise rate, is applied to test thermal conductivity and special heat of specimens. The temperature of the system and time are linear. Furthermore, the temperature rise rate is the same. The test instrument is ZKY-BRDR and Technology Co., Ltd., China) in this experiment. The temperature difference and temperature rise rate

7 of specimens were tested. The thermal conductivity is as follows.

$$
\lambda=\frac{q_{c} R}{2 \Delta t}
$$

8 where $\lambda$ is thermal conductivity, $\Delta t$ is the temperature difference between the heating surface and the

9 central surface under the quasi steady state, $R$ is the sample thickness ( $R$ is $10 \mathrm{~mm}$ in this experiment),

10 and $q_{c}$ is the density of heat flow rate.

$$
\Delta t=\frac{V_{t}}{0.04}(k)
$$

11 where $V_{t}$ is named as the thermoelectric potential between the heating surface and the central surface.

$$
q_{c}=\frac{V^{2}}{2 F r}\left(w / m^{2}\right)
$$

12 where $V$ is heating voltage of two parallel heater., $F$ is heating area after edge is revised ( $F=$

$13 A \times 0.09 \mathrm{~m} \times 0.09 \mathrm{~m}$, where $A$ is corrected coefficient), and $r(110 \Omega)$ is resistance of each heater.

$$
c=\frac{q_{c}}{\rho R \frac{d t}{d \tau}}
$$

14

where $c$ is special heat, $\rho$ is density of specimen, which is equal to $2.4 \mathrm{~g} / \mathrm{cm}^{3}$.

$$
\frac{d t}{d \tau}=\frac{\Delta V}{60 \times 0.04}(k / s)
$$

15 where $\frac{d t}{d \tau}$ is temperature rise rate of specimen under quasi steady condition, $\Delta V$ is temperature rise 
1

2 content of NGPs. Therefore, the hardness of cementitious composite also increases as the content of

of thermoelectric potential in each minute.

\subsubsection{Test for electromagnetic wave absorbing performance}

PNA-X Network analyzer N5244A (Agilent Technologies Co., Ltd., America) was used to test the electromagnetic wave absorbing performance of cementitious composites filled with NGPs.

The experimental result is the mean value of three specimens in each group.

\section{Results and Discussions}

\subsection{Hardness of cementitious composites filled with NGPs}

The hardness cloud images of cementitious composite at $0 \%, 1 \%$ and $5 \%$ loading of NGPs are shown in Figs. 1a, b and c, respectively. The average hardness of cementitious composite at different content of NGPs can be calculated and illustrated in Fig. 2. It can be seen from Fig. 2 that the hardness of cementitious composite shows an increase trend with the increasing content of NGPs. Furthermore, the hardness firstly increases and then remains stable with increasing penetration depth. The hardness of pure cementitious composites is about $4.79 \mathrm{GPa}$, which is close to hardness of the hardened cement paste. The hardness of cementitious composite with $1 \%$ loading of NGPs increases by $75.57 \%$ compared with plain cementitious composites. The hardness of cementitious composite with 5\% loading of NGPs represents a 1.5-fold increase over plain cementitious composites. It can be inferred that the abrasion resistance performance of cementitious composite can be enhanced due to the addition of NGPs.

As we know, the distribution density of NGPs in the cementitious composite increases with the increasing content of NGPs. Thus reinforcing effect becomes more obvious. Besides, due to the nano-filling effect of NGPs, the density of cementitious composite is improved with the increasing NGPs increases. 


\subsection{Abrasion resistance performance of cementitious composites filled with NGPs}

Fig. 3 shows the relationship between the content of NGPs and the abrasive loss per unit area of cementitious composite. The abrasive loss per unit area of cementitious composite filled with NGPs shows obviously decreasing trend with the increasing content of NGPs. The abrasive loss per unit area of cementitious composite with 5\% loading of NGPs represents a $70.88 \%$ decrease over pure cementitious composites. Therefore, NGPs play a distinct role in improving the abrasion resistance performance of cementitious composite.

Fig. 4 displays the relationship between the content of NGPs and the abrasion depth of cementitious composite. The abrasion depth of specimen declines sharply as the content of NGPs increases. The abrasion depth of cementitious composite at 5\% loading of NGPs is only $3 \mathrm{~mm}$ and decreases by $70.96 \%$ compared with plain cementitious composites. It illustrates further that the NGPs can improve the abrasion resistance performance of cementitious composite. The composites with higher hardness and abrasion resistance are more resistant to pressure and wear.

The addition of NGPs can improve the abrasion resistance property of cementitious composites, which can be attributed to the self-lubricating property and high hardness of NGPs. As we know, the graphite is a typical lamellar compound. Fig. 5 shows the overview microstructures of NGPs. It can be seen that NGPs are quite well dispersed and cement matrix become denser with increasing NGPs content. The NGPs can enhance the friction-bearing capacity of the composite through modifying the hardness of the composites. In other word, the high hardness can better resist abrasion. The clear laminated structure of NGPs can be seen in SEM images. Sliding relatively between the multi-layer structures of carbon-carbon hexagonal plane endows the graphite with lubricating function. As for lubricant mechanism of cementitious composites filled with NGPs, there are two main reasons. On the one hand it 
1 is not a chemical bond between the NGPs composite fillers and cementitious composites. Therefore, lubricant molecules and polymer molecules are separated from each other under the influence of frictional heat. Then nanoparticles of NGPs are pushed to the frictional surface. At the same time it is just filled with the pits of frictional surface, which is equivalent to the surface-repairing function. On the other hand, NGPs, which act as the lubricant, will give priority to transfer into the grinding surface in the process of friction and form lubricating film on the frictional surface. This behavior gives rise to a decreasing trend in abrasion between friction pair and the cementitious composites filled with NGPs. On the basis of the above analysis and discussion, it is found that the filling effect of nanoparticles becomes better and the area of the lubricating film in the frictional surface becomes grouter with the increasing content of NGPs in cementitious composites in a certain dosage range. In other word, the abrasion loss of the cementitious composites will reduce.

\subsection{Damping performance of cementitious composites filled with NGPs}

Fig. 6 expresses the free vibration time-history decay curves of the cementitious composites filled with different contents of NGPs. As shown in Fig. 6, the acceleration amplitude attenuates roughly between $0-0.04 \mathrm{~s}$ after incentive action. Through analysis and comparison, it can be seen that the bigger dosage of NGPs is, the greater attenuation rate of acceleration is. Fig. 7 shows the damping ratio of cementitious composites filled with NGPs at different concentrations. It can be seen from Fig. 7 that the damping ratio of cementitious composite is directly proportional to the amount of NGPs. When the NGPs are not added, the damping ratio of pure cementitious composites is 0.01 . However, the damping ratio of cementitious composite increases by $4.7 \%$ when the NGPs content is 1 vol. $\%$. The damping ratio of cementitious composites increases by $20 \%$ as the content is 5 vol. $\%$. This also indirectly proves that NGPs have a potential to improve the damping property of cementitious composite. The improvement of 
1 damping is beneficial to vibration attenuation. References [43-46] have reported that the carbon nanotubes feature significant reinforcement to the damping properties of the polymer-matrix and ceramic-matrix composites. The reinforcement of NGPs to damping properties of the cementitious composites is different from that in above references because the matrix, filler and structure of the composites in the references 43-46are obviously different from those in this paper. In addition, the improvement of carbon nanotubes on the damping properties of the cementitious composite can reach $18.3 \%$ [47], which is lower than $20 \%$ of enhancement of NGPs to the damping performance of the cementitious composites in this paper.

In generally, introducing the material damping can make vibrational energy of structure convert irreversibly into other formal energy, which reaches the purpose of dissipating vibrational energy through internal system of material. The reasons for composite fillers can improve the damping property of the cementitious composites can be divided into two types. Firstly, the addition of composite fillers make the cementitious composites create inevitably some defects, such as dislocations, phase boundaries, grain boundaries, weak interface. These defects can dissipate vibrational energy. Secondly, the viscoelastic admixtures can provide damping and dissipate vibrational energy. The types of dissipating energy of NGPs mainly have three kinds.

(1) The dislocation slip in NGPs own layer consumes vibrational energy. The lamellar structure of graphite is hexagonal plane lattice unit connected by covalent bond. The interlayer relies on the connection between delocalized $\pi$ bonds that is similar to metal bond and van der Waals forces. Because of the small interlayer binding force and large gaps between the layers, it is easy to create relative slip for NGPs, which can dissipate vibrational energy and cause high internal friction.

(2) Viscous friction between NGPs composite fillers and the cementitious composites consumes 
1 vibrational energy. The cementitious composites appear diversity phase boundaries and crystal interfaces when NGPs is combined with the cementitious composites. In addition, a large number of diversity interfaces increase frictional energy of cementitious composites increase. Under the action of shear stress, deformation between the two interfaces is short of coordination, which leads to interface friction.

5 Furthermore, these interfaces also make contribute to dislocation damping.

6 (3) Contact friction between the hydration productions inside the cement matrix and NGPs fillers

7 consumes vibrational energy.

8

\subsection{Thermal conductivity performance of cementitious composites filled with NGPs}

A plot of the thermal conductivity of cementitious composites filled with NGPs is presented in Fig. 8.

The specific heat is chosen to represent the thermal conductivity performance of cementitious composites filled with NGPs in Fig. 9. The thermal conductivity of cementitious composites filled with NGPs grows with increasing content of the NGPs. The thermal conductivity of cementitious composites at 1 vol. \% loading of NGPs reaches $1.22 \mathrm{~W} / \mathrm{m} . \mathrm{K}$, which represents an $11 \%$ increase over plain cementitious composites. It is amazing that the thermal conductivity of cementitious composite is twice as much as that of plain cementitious composites when the content of NGPs is 5\%. The specific heat of cementitious composites is negatively correlated to the dosage of NGPs. Compared with cementitious composites without NGPs, the specific heat of cementitious composite at $1 \%$ loading of NGPs decreases by $5.88 \%$. The specific heat of cementitious composites filled with 5\% loading of NGPs is $17.7 \%$ smaller than that of pure cementitious composites. The small specific heat indicates the large temperature rise rate, which implies further the better thermal conductivity property. In contrast, the large thermal conductivity indicates the good thermal conductivity property. Thus, it is inferred that NGPs can enhance the thermal conductivity performance of the cementitious composite. The increasing thermal conductivity is 
1 beneficial for transmitting and releasing heat of hydration, and maintaining the uniformity of the temperature and decreasing the thermal stress inside the composites. flow. It is attribute the outstanding thermal conductivity property of this material to a favorable combination of the high aspect ratio, stable crystal structure, ordered carbon atom layer and low thermal interface resistance of the NGPs. In the process of thermal vibration, the effective transmission of phonons reflects the good thermal conductivity of crystals. Hence, the high thermal conductivity of NGPs can be explained from the following 4 aspects.

9 (1) The carbon bond of graphene has good flexibility [48] and its energy is very high [49]. When external force is applied to the graphene, the surface of carbon atoms may be bent and deformed. In other word,

11 the carbon atoms do not need to rearrange to adapt to the changes of the outside world, which maintain

12 the stability of the structure. At room temperature, when the carriers motion in the crystal, they are mainly

13 suffered from scattering of phonon. However, the stable lattice structure can reduce the scattering in the phonon transmission.

15 (2) The ordered carbon atom layer within the NGPs provides a highly efficient channel of heat transfer,

16 which promotes the phonon transmission between the NGPs and the cementitious composites [50].

17 (3) Compared with other fillers, such as spherical or cylindrical shape, the sheet thermal-conductive fillers have more advantages. Because the close contact is formed between the adjacent sheet fillers and

19 the contact area of the sheet filler is larger, the interfacial thermal resistance can be reduced and the

20 phonon transmission can be improved $[50,51]$.

21 (4) Compared with the cementitious material, the nanoparticles can substantially improve the thermal conductivity of the material. It is because that the nanoparticles provide heat-transfer carrier, so as to 
enhance the electron mobility [52].

\subsection{Electromagnetic wave absorbing performance of cementitious composites filled with NGPs}

The reflectivity of the electromagnetic wave of the cementitious composites with different NGP

contents is tested in the $1-18 \mathrm{GHz}$ frequency band, as shown in Fig. 10. It is easy to find that the trend of the reflectivity of cementitious composites in three groups is basically similar. The only difference lies in the amplitude of the change. The two wave valleys appear successively in the low frequency band $(1-8 \mathrm{GHz})$. The first wave valley appears in the vicinity of $3 \mathrm{GHz}$. The reflectivities of pure cementitious composites and cementitious composite with $1 \%$ loading of NGPs are about $-1.25 \mathrm{GHz}$. However, reflectivity of cementitious composite with $5 \%$ loading of NGPs is $-1.37 \mathrm{GHz}$. The reflectivity of cementitious composite achieves the maximum value of the entire frequency band $(1-18 \mathrm{GHz})$ in the vicinity of $8 \mathrm{~Hz}$. It is indicated that the electromagnetic wave absorbing performance of cementitious composites reaches the best in the vicinity of $8 \mathrm{GHz}$. At the same frequency, the reflectivity of the cementitious composite decreases with the increasing content of NGPs. Compared with the reflectivity of blank cementitious composites, the reflectivity of cementitious composite with $1 \%$ dosage of NGPs is $-3.96 \mathrm{GHz}$ and reduces by $12 \%$. The reflectivity of cementitious composite with $5 \%$ dosage of NGPs reduces by $38 \%$ (reach $-4.87 \mathrm{GHz}$ ). At the high frequency band $(8-18 \mathrm{GHz})$, the trend of reflectivity is similar to that of the composites at the low frequency band. The reflectivity changes with the frequency, but the change amplitude becomes smaller. In the $14-18 \mathrm{GHz}$ band, the electromagnetic wave absorbing curve is basically in the platform. The reflectivity of cementitious composite reaches the valley value in near $13 \mathrm{GHz}$. Unlike the reflectivity of composite at low frequency band, the reflectivity of the composite even increases with the increasing dosage of NGPs at high frequency band. The electrical resistance of the cementitious composites decreases with the increasing content of NGPs, which leads to the reduction 
1 of the electromagnetic wave absorbing property of the composite [53]. However, there is strong electromagnetic wave absorbing property of cementitious composite with 5\% loading of NGPs in vicinity of $8 \mathrm{GHz}$. Therefore, we believe that NGPs have some good influence on electromagnetic wave absorbing of cementitious composite. The good wave absorbing performance means the cementitious composites is beneficial for reducing electromagnetic pollution, enhancing military camouflage, and preventing information leakage.

If cementitious composite is expected to have good electromagnetic wave absorbing property, first of all, it should have good electromagnetic wave impedance matching. On the one hand, it should reduce reflection of electromagnetic wave and enhance incidence of electromagnetic wave. On the other hand it should enhance electromagnetic wave absorbing in the absorbing layer. Secondly, cementitious composites should meet the attenuation characteristics. Composites with high electromagnetic loss can absorb and attenuate the electromagnetic wave. Only in this way it can improve the electromagnetic wave absorbing effect of the cementitious composites layer. The absorbing mechanism of the composites is originated from the dielectric property of NGPs and its unique structure of carbon-carbon hexagonal plane. The graphite with high electrical conductivity is a resistance-type absorbent and have a high dielectric loss angle tangent. Thus the graphite relies on attenuation of electronic polarization in the surface of medium or interfacial polarization to absorb electromagnetic wave. NGPs that are dispersed in the cementitious composites form countless scatter points. The electromagnetic wave consumes energy by multiple scattering. The effect that electromagnetic wave reflects and refracts repeatedly in the dihedral angle of graphite sheet combines with the dielectric loss of the graphite, so as to achieve the function of electromagnetic wave absorbing. In addition, there is transverse vibration of the lattice in layered structure of monolithic graphite. The transverse vibration can interact with transverse electromagnetic 
1 wave. When the frequency of electromagnetic wave is the same as that of graphite lattice transverse vibration, lattice vibration may create resonant absorption of electromagnetic wave.

3

\section{Conclusions}

Cementitious composites filled with NGPs at levels of $0,1 \%$, and $5 \%$ were fabricated. The hardness, abrasion resistance, damping, thermal conductivity and electromagnetic wave absorbing properties of cementitious composites were investigated.

(1) The hardness of NGPs is higher than that of cementitious composites. Moreover the hardness of cementitious composite increases as NGPs increases. When the content of NGPs is $5 \%$, the hardness of cementitious composite grows by about 1.5 -fold compared with pure cementitious composites.

(2) The abrasive loss per unit area of cementitious composites with NGPs at levels of $0 \%, 1 \%, 5 \%$ decreases from $26 \mathrm{~kg} / \mathrm{m}^{2}$ to $20.993 \mathrm{~kg} / \mathrm{m}^{2}$ and then downed to $7.573 \mathrm{~kg} / \mathrm{m}^{2}$, which represents a $19.5 \%$ and $70.88 \%$ decrease over pure cementitious composites, respectively. The abrasion depth of cementitious composites with NGPs at loading of $0 \%, 1 \%, 5 \%$ decreases from $10.33 \mathrm{~mm}$ to $8.33 \mathrm{~mm}$ and then downed to $3 \mathrm{~mm}$, which presents a $24 \%$ and $70.96 \%$ decrease over pure cementitious composites, respectively. The addition of NGPs can enhance abrasion resistance performance of cementitious composites from above two sets of data. On the one hand the hardness of single layer nanographite is very large. On the other hand the graphite is a kind of good lubrication material. The lubricating film formed between the cement matrix and NGPs in the frictional process can reduce the abrasion of the cementitious composites. The nanoparticles will be filled with pits of frictional surface, which also has an anti-friction influence on cementitious composites.

(3) Compared with plain cementitious composites, the damping ratio of cementitious composites filled with NGPs at level of $1 \%$ and $5 \%$ increased by $4.7 \%$ and $20 \%$ respectively. The damping property of 
1 cementitious composites filled with NGPs mainly comes from dislocation slip in NGPs own layer,

2 viscous friction between NGPs composite fillers and the cementitious composites and contact friction

3 between the productions inside the cement matrix.

4 (4) Compared with plain cementitious composites, thermal conductivity of cementitious composite at and $5 \%$ levels of NGPs increases from $1.22 \mathrm{~W} / \mathrm{m} . \mathrm{K}$ to $1.94 \mathrm{~W} / \mathrm{m} . \mathrm{K}$, which presents an of $11.3 \%$ and $77 \%$ increase respectively. The specific heat of cementitious composites decreases with increasing content of

7 NGPs. The flexible carbon bond provides NGPs with the stability structural, which is helpful for phonon transmission. Moreover, the ordered carbon atom layer of NGPs and sheet thermal-conductive fillers both

9 improve phonon transmission and accelerate heat conduction.

10 (5) Electromagnetic wave reflectivity of cementitious composites at 5\% loading of NGPs reaches the

11 minimum value $(-5 \mathrm{~dB})$ in the vicinity of $8 \mathrm{~Hz}$. The absolute value of reflectivity increases by $38 \%$

12 compared with plain cementitious composites. It indicated that NGPs have an obvious influence on

13 electromagnetic wave absorbing performance of cementitious composites. The high dielectric loss angle tangent and resonance absorption make NGPs have good electromagnetic wave absorbing property. NGPs are promising composite fillers to develop multifunctional cementitious composites, which have wide application in various fields of civil engineering, such as pavement, port, buildings for earthquake resistance, large volume of concrete and military engineering.

\section{Acknowledgments}

19 The authors thank the funding supported from the National Science Foundation of China (51578110 and

20 51428801).

\section{$21 \quad$ References}

22 [1] Han B, Sun S, Ding S, et al. Review of nanocarbon-engineered multifunctional cementitious 
1 composites. Composites Part A: Applied Science and Manufacturing. 2015, 70: 69-81.

2 [2] Hang S, Xu R, Liu F, et al. Development and application of cement-based functional composite.

3 Silicate Bulletin. 2003(04): 58-63.

4 [3] Luo J. Fabrication and functional properties of multi-walled carbon nanotube/cement composites.

5 Harbin Institute of Technology, Thesis of doctor degree, 2009.

6 [4] Ma P C, Siddiqui N A, Marom G, et al. Dispersion and functionalization of carbon nanotubes for

7 polymer-based nanocomposites: a review. Compos Part A: Applied Science and Manufacturing.

$8 \quad 2010 ; 41(10): 1345-67$.

9 [5] Chyad F A. The effects of metastable zirconia on the properties of ordinary Portland cement.

10 Dissertation for the Doctoral Degree, University of Bradford, England. 1989.

11 [6] Sanchez F and Sobolev K. Nanotechnology in concrete-a review. Construction and Building Materials.

$12 \quad 2010,24(11): 2060-2071$.

13 [7] Chen $\mathrm{J}$ and Poon C. Photocatalytic construction and building materials: from fundamentals to applications. Building and Environment. 2009, 44(9): 1899-1906.

15 [8]D'Alessandro A, Ubertini F, Materazzi A L, et al. Electromechanical modelling of a new class of 16 nanocomposite cement-based sensors for structural health monitoring. Structural Health Monitoring, 2014, 17 14(2).

18 [9] Ubertini F, Laflamme S, Ceylan H, et al. Novel nanocomposite technologies for dynamic monitoring 19 of structures: a comparison between cement-based embeddable and soft elastomeric surface sensors.

20 Smart Materials Structures, 2014, 23(4):12p.

21 [10] Meyers F N, Loh K J, Dodds J S, et al. Active sensing and damage detection using piezoelectric zinc oxide-based nanocomposites. Nanotechnology, 2013, 24(18):185501-185501. 
1 [11] Du H, Pang S D. Enhancement of barrier properties of cement mortar with graphene nanoplatelet.

2 Cement and Concrete Research, 2015, 76:10-19.

3 [12] Peyvandi A, Soroushian P, Jahangirnejad S. Enhancement of the structural efficiency and

4 performance of concrete pipes through fiber reinforcement. Construction and Building Materials, 2013,

$5 \quad 45(45): 36-44$.

6 [13] Du H, Gao H J, Pang S D. Improvement in concrete resistance against water and chloride ingress by

7 adding graphene nanoplatelet. Cement and Concrete Research, 2016, 83:114-123.

8 [14] Saafi M, Tang L, Fung J, et al. Graphene/fly ash geopolymeric composites as self-sensing structural

9 materials. Smart Materials and Structures, 2014, 23(6):182-186.

10 [15] Le J L, Du H, Pang S D. Use of 2D graphene nanoplatelets (GNP) in cement composites for

11 structural health evaluation. Composites Part B: Engineering, 2014, 67:555-563.

12 [16] Ansari S, Giannelis E P. Functionalized graphene sheet-poly (vinylidene fluoride) conductive

13 nanocomposites. Journal of Polymer Science Part B: Polymer Physics. 2009, 47(9): 888-897.

14 [17] Rafiee M A. Graphene-based composite materials. Nature, 2006, 442(7100):282-286.

15 [18] Sedaghat A, Ram M K, Zayed A, et al. Investigation of physical properties of graphene-cement

16 composite for structural applications. Open Journal of Composite Materials. 2014, 04(01): 12-21.

17 [19] Tong T, Fan Z, Liu Q, et al. Investigation of the effects of graphene and graphene oxide nanoplatelets on the micro- and macro-properties of cementitious materials. Construction \& Building Materials, 2016, 106:102-114.

[20] Cao M, Zhang H, Zhang C. Effect of graphene on mechanical properties of cement mortars. Journal of Central South University, 2016, 23(4):919-925. 
cementitious composites. Construction \& Building Materials, 2016, 120:457-464. mWpd0ystYb1CfsNHf0AmyZ5x4Ze1ALbp0o_. liquid-phase-sintered SiC containing graphite nanodispersoids. Journal of the European Ceramic Society. 2014, 34(10): 2597-2602. situ-toughened silicon carbide. Journal of the American Ceramic Society. 2005, 88(12): 3531-3534. [25] Borrero-López O, Ortiz A L, Guiberteau F, et al. Sliding-wear-resistant liquid-phase-sintered SiC processed using $\alpha$-SiC starting powders. Journal of the American Ceramic Society. 2007, 90(2): 541-545. [26] Du J, Li Y, Zheng X. Effect of nano-graphite on friction performance of Cu-based friction material. Advanced Materials Research. 2011, 284-286: 905-908. [27] Lao X. Research on friction properties of graphene. University of Electronic Science of Technology of China, Thesis of master degree, 2008. composites. Shangdong University of Science and Technology, Thesis of doctor degree ,2005. [29] Cheng H F, Huang X M, Wei J N, et al. Damping behavior of graphite-reinforced Al matrix composite . Mining and Metallurgical Engineering. 2002, 22(2): 103-106. [30] Liu X, Tang S, Li X, et al. Effects of nano-graphite on the damping properties of aluminum matrix composite materials. Mechanical Science and Technology. 2006(12): 1428-1430. commercially pure aluminum matrix composites. Materials for Mechanical Engineering. 2010(11): 88-90. 
1 [32] Muthusamy S, Wang S, Chung D D L. Unprecedented vibration damping with high values of loss modulus and loss tangent, exhibited by cement-matrix graphite network composite. Carbon. 2010, 48(5):

3
1457-1464.

[33] Faugeras C, Faugeras B, Orlita M, et al. Thermal conductivity of graphene in corbino membrane geometry. ACS Nano. 2010, 4(4): 1889-1892.

[34] Chandrasekaran S, Seidel C, Schulte K. Preparation and characterization of graphite nano-platelet (GNP)/epoxy nano-composite: mechanical, electrical and thermal properties. European Polymer Journal. 2013, 49(12): 3878-3888.

[35] Raza M A, Westwood A, Brown A, et al. Characterisation of graphite nanoplatelets and the physical properties of graphite nanoplatelet/silicone composites for thermal interface applications. Carbon. 2011, 49(13): 4269-4279.

[36] Yu A, Ramesh P, Itkis M E, et al. Graphite nanoplatelet-epoxy composite thermal interface materials. The Journal of Physical Chemistry C. 2007, 111(21): 7565-7569.

[37] Guo A. Research on properties of radar wave absorption of cement-based composite materials.

Harbin Institute of Technology, Thesis of doctor degree, 2009.

[38] Ji Z, Han B, Hou G, et al. Electromagnetic wave absorbing properties of cement-based expanded perlite mortar with graphite. Materials Science and Technology. 2011(02): 15-18.

[39] Han B, Ji Z, Zhang Z, et al. Research on absorbing properties of cement-based coating material. Material Report. 2009(23): 370-373.

[40] Shafieizadegan-Esfahani A R, Katbab A A, Dehkhoda P, et al. Preparation and characterization of foamed polyurethane/silicone rubber/graphite nanocomposite as radio frequency wave absorbing material: The role of interfacial compatibilization. Composites Science and Technology, 2012, 72(3):382-389. 
1 [41] Shafieizadegan-Esfahani A R, Katbab A A, Pakdaman A R, et al. Electrically conductive foamed

2 polyurethane/silicone rubber/graphite nanocomposites as radio frequency wave absorbing material: The

3 role of foam structure. Polymer Composites. 2012, 33(3): 397-403.

4 [42] Wang G, Zhang X, Wei Y, et al. Polymer composites with enhanced wave absorption properties

5 based on modified graphite and polyvinylidene fluoride. Journal of Materials Chemistry A, 2013,

$6 \quad 1(24): 7031-7036$.

7 [43] Koratkar N, Wei B Q, Ajayan P M. Carbon nanotube films for damping applications. Advanced

8 Materials, 2002, 14(13-14):997-1000.

9 [44] Khan S U, Li C Y, Siddiqui N A, et al. Vibration damping characteristics of carbon fiber-reinforced

10 composites containing multi-walled carbon nanotubes. Composites Science and Technology, 2011,

11 71(12):1486-1494.

12 [45] Balani K, Agarwal A. Damping behavior of carbon nanotube reinforced aluminum oxide coatings by

13 nanomechanical dynamic modulus mapping. Journal of Applied Physics, 2008, 104(6):063517-063517-6.

14 [46] Her S C, Lai C Y. Dynamic behavior of nanocomposites reinforced with multi-walled carbon

15 nanotubes (MWCNTs). Materials, 2013, 6(6):2274-2284.

16 [47] Li W W, Ji W M, Liu Y, et al. Damping property of a cement-based material containing carbon

17 nanotube. Journal of Nanomaterials, 2015, 2015(23).

18 [48] Hong Y, Li L, Zeng X C, et al. Tuning thermal contact conductance at graphene-copper interface via

19 surface nanoengineering. Nanoscale, 2015, 7(14):6286-94.

20 [49] Liu C, Yu Z, Neff D, et al. Graphene-based supercapacitor with an ultrahigh energy density. Nano

21 Letters, 2010, 10(12):4863-4868.

22 [50] Min C, Yu D, Cao J, et al. A graphite nanoplatelet/epoxy composite with high dielectric constant and 
1 high thermal conductivity. Carbon, 2013, 55(2):116-125.

2 [51] Kalaitzidou K, Fukushima H, Drzal L T. Multifunctional polypropylene composites produced by

3 incorporation of exfoliated graphite nanoplatelets. Carbon, 2007, 45(7):1446-1452.

4 [52] Lin J. Preparation and investigation of nano-carbon composite materials. Guangdong University of

5 Technology, Thesis of doctor degree, 2014.

6 [53] Itoh M, Liu J R, Horikawa T, et al. Electromagnetic wave absorption properties of nanocomposite

7 powders derived from intermetallic compounds and amorphous carbon. Journal of Alloys and Compounds,

$8 \quad 2006$, s 408-412(408):1400-1403.

9 


\section{$1 \quad$ Figure captions}

2 Fig.1 Nano-indentation hardness cloud images of cementitious composites filled with: a) $0 \%$ NGPs; b) $1 \%$

$3 \quad$ NGPs; c) 5\% NGPs

4 Fig. 2 Hardness of cementitious composites filled with NGPs

5 Fig. 3 Abrasive loss per unit area of cementitious composites filled with NGPs

6 Fig. 4 Abrasion depth of cementitious composites filled with NGPs

7 Fig. 5 SEM images of cementitious composites filled with: a) $0 \%$ NGPs; b) $1 \%$ NGPs; c) 5\% NGPs

8 Fig. 6 Free vibration response time-history decay curve and local amplification of cementitious

9 composites filled with NGPs

10 Fig. 7 Damping ratio of cementitious composites filled with NGPs

11 Fig. 8 Thermal conductivity of cementitious composites with NGPs

12 Fig. 9 Specific heat of cementitious composites filled with NGPs

13 Fig. 10 Reflectivity of cementitious composites filled with NGPs 
a)

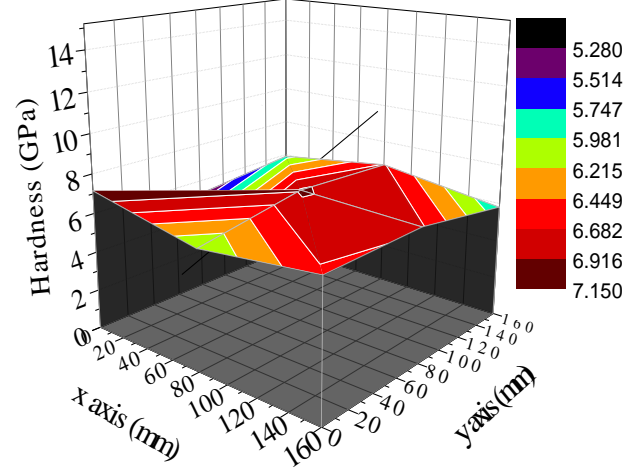

b)

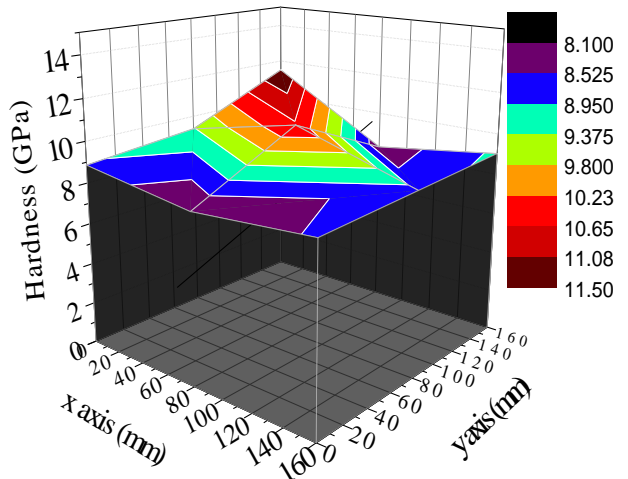

c)

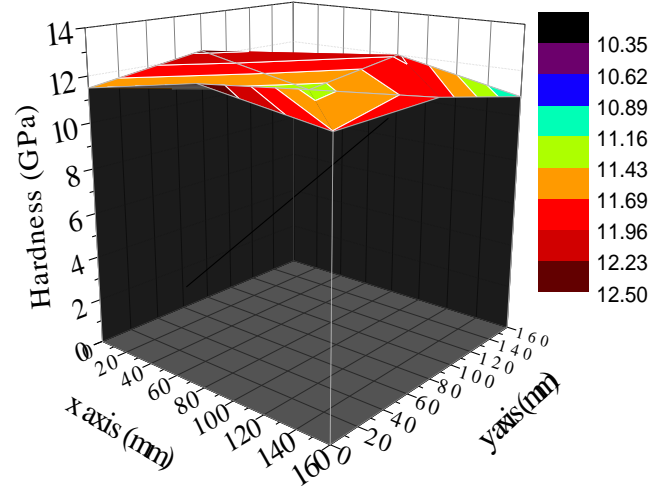

Fig. 1 Nano-indentation hardness cloud images of cementitious composites filled with: a) $0 \%$ NGPs; b) $1 \%$ NGPs; c) $5 \%$ NGPs
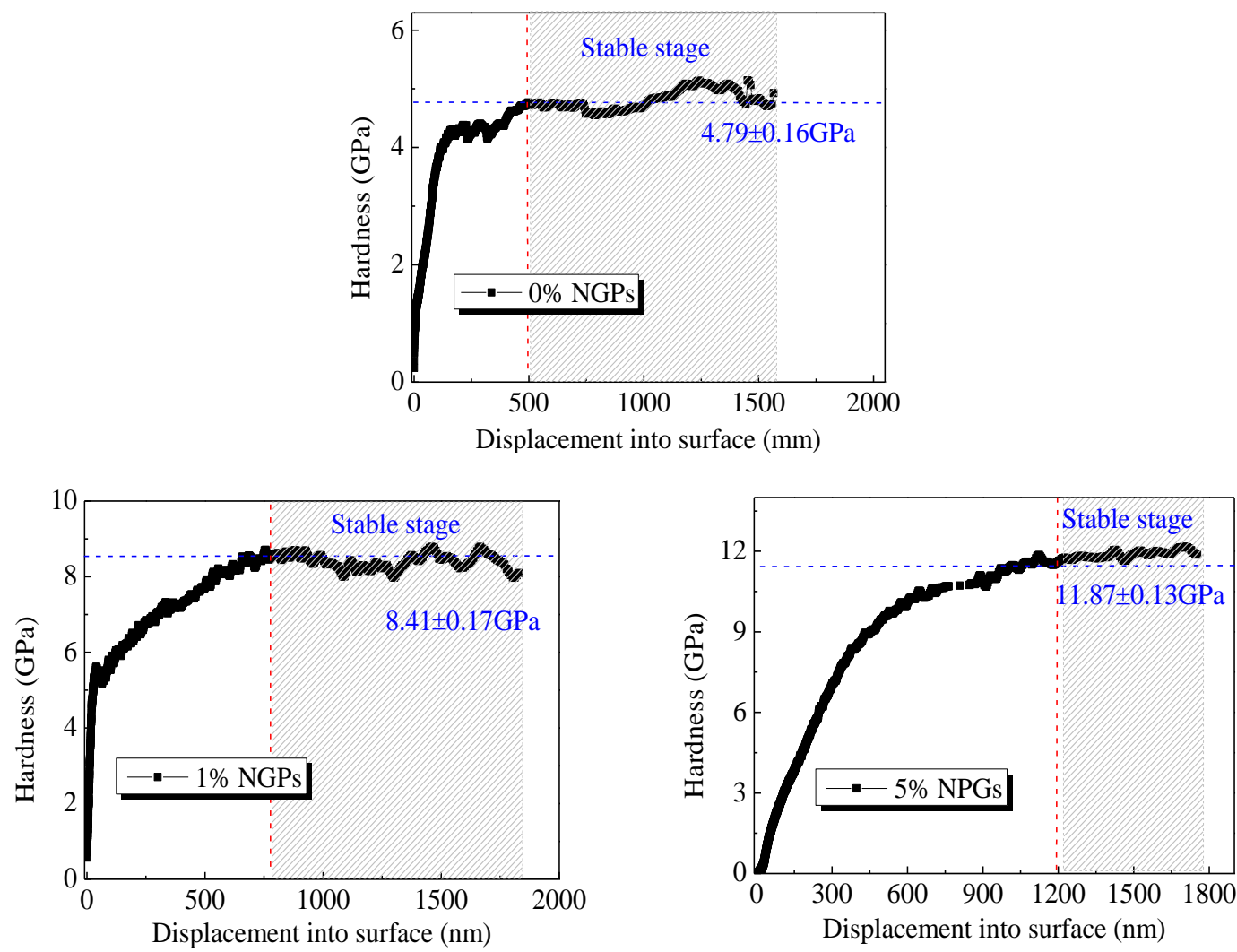

Fig. 2 Hardness of cementitious composites filled with NGPs 


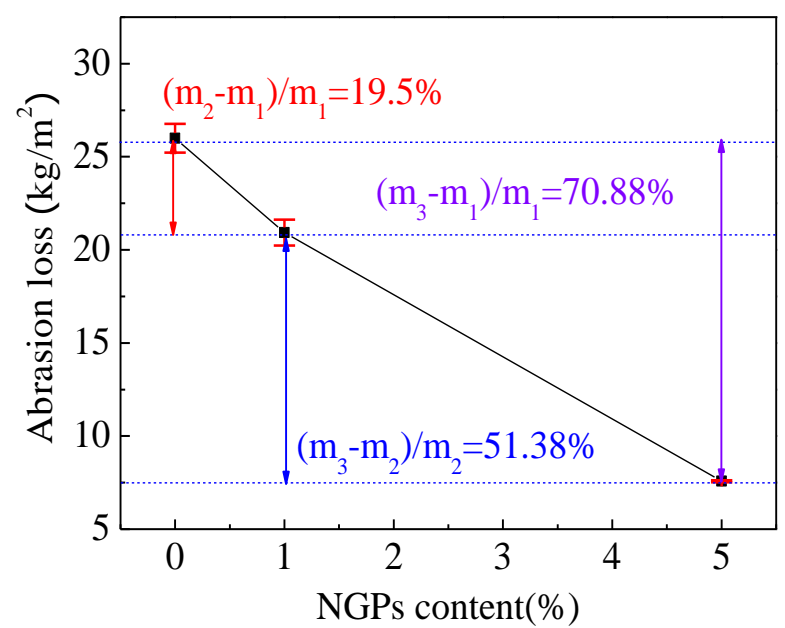

Fig. 3 Abrasive loss per unit area of cementitious composites filled with NGPs

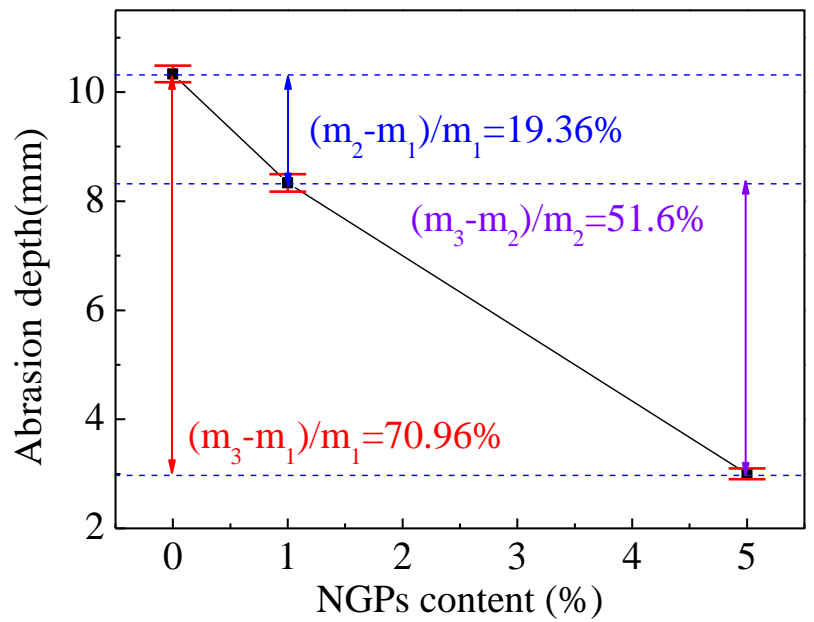

Fig. 4 Abrasion depth of cementitious composites filled with NGPs

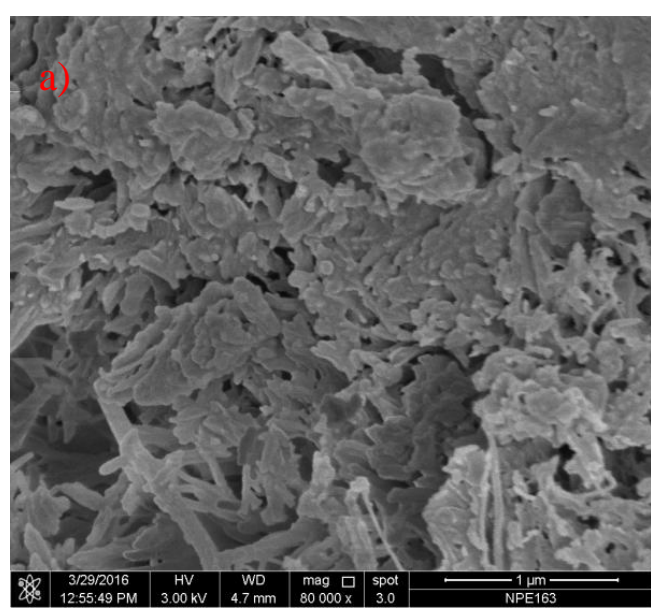



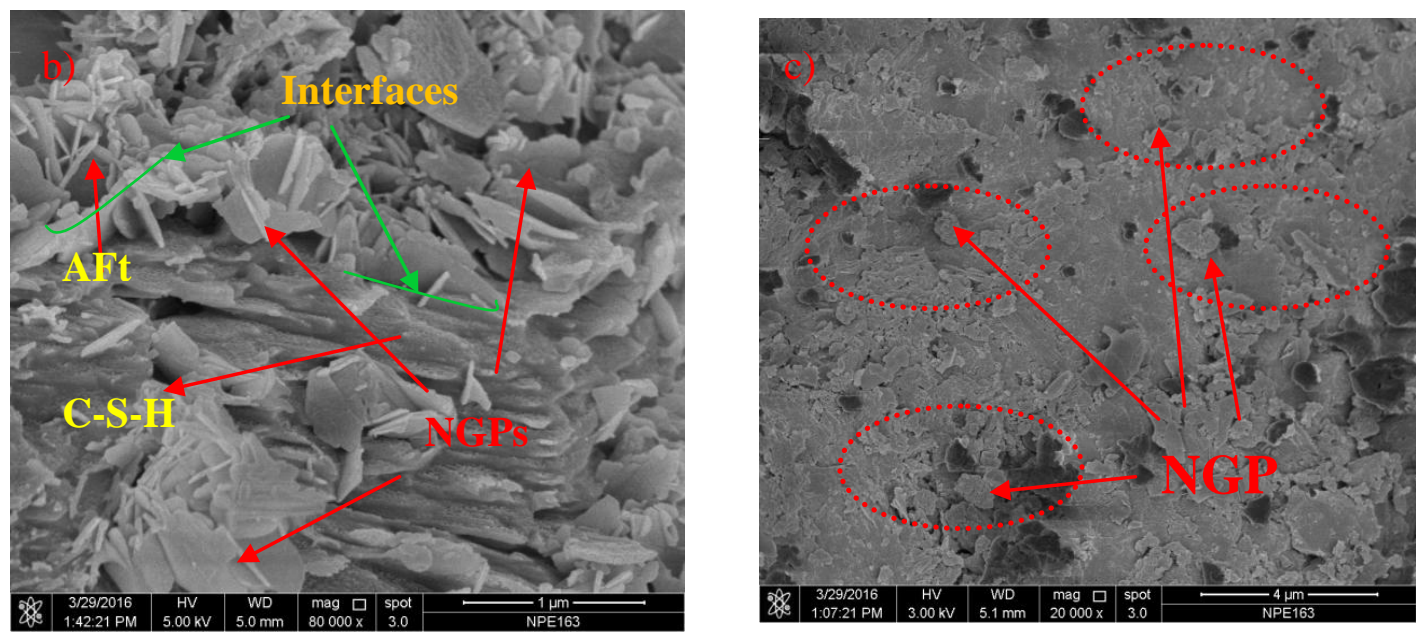

Fig. 5 SEM images of cementitious composites filled with: a) $0 \%$ NGPs; b) $1 \%$ NGPs; c) $5 \%$ NGPs 1
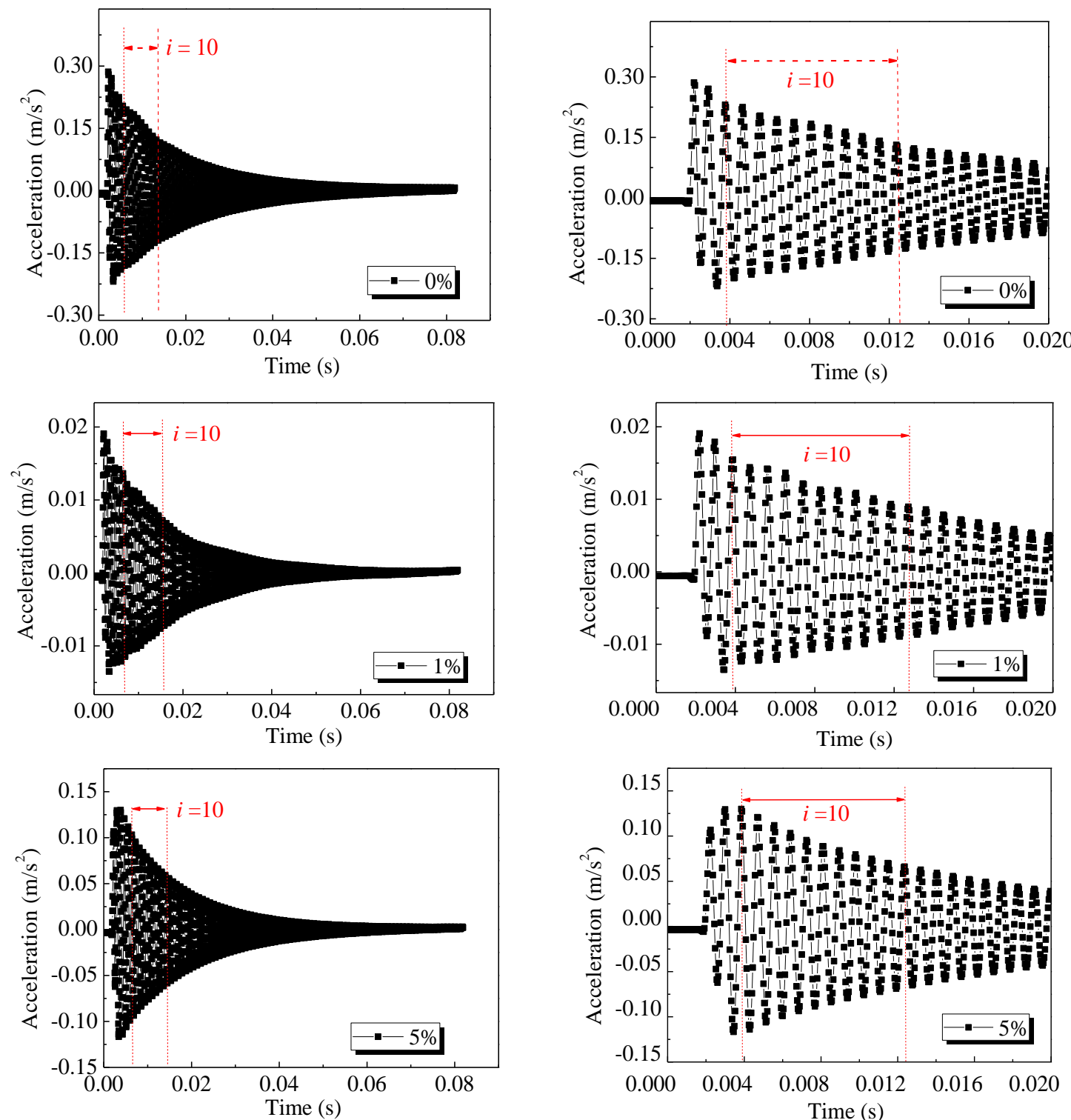

Fig. 6 Free vibration response time-history decay curve and local amplification of cementitious composites filled with NGPs 


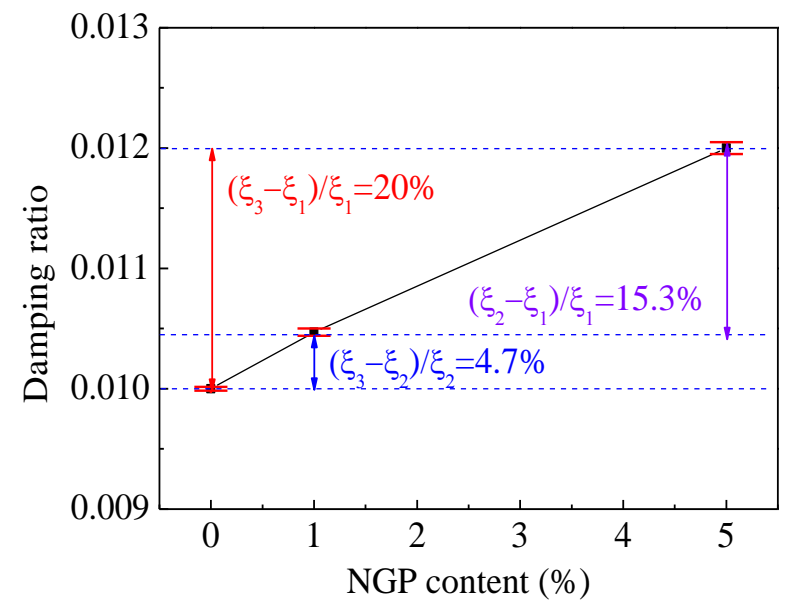

Fig. 7 Damping ratio of cementitious composites filled with NGPs

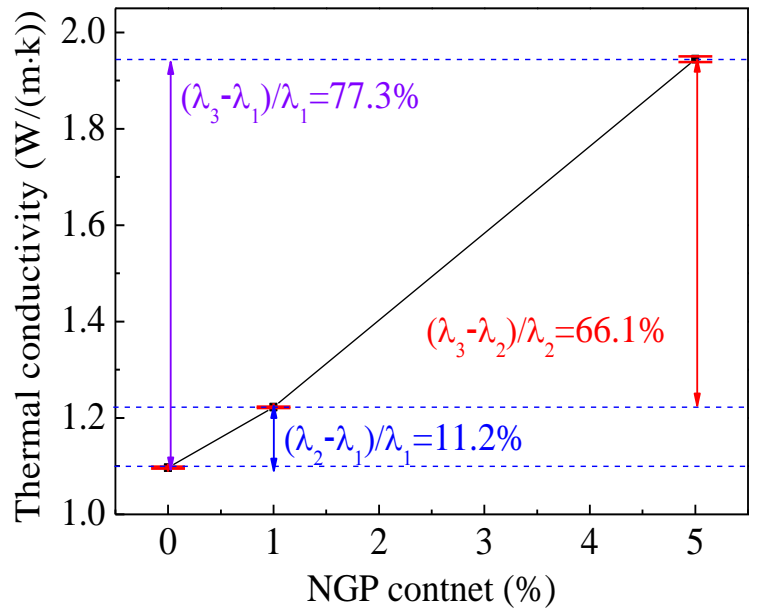

Fig. 8 Thermal conductivity of cementitious composites with NGPs

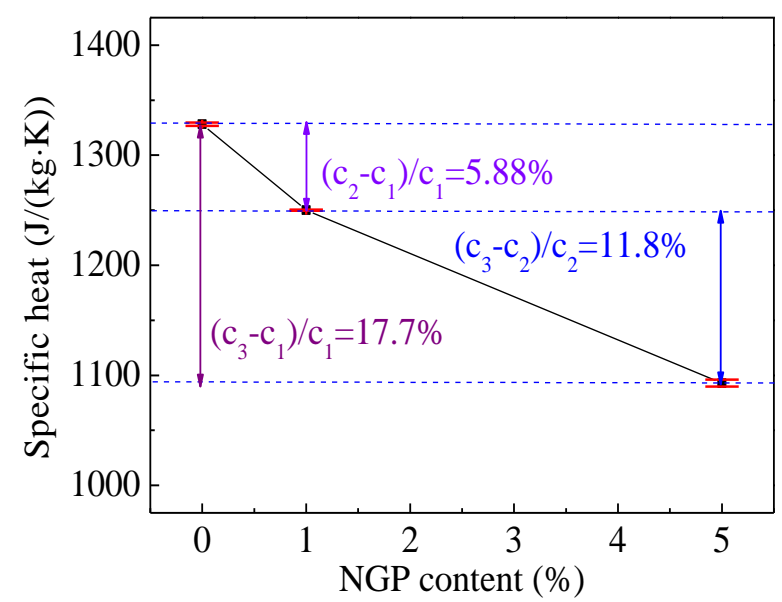

Fig. 9 Specific heat of cementitious composites filled with NGPs 


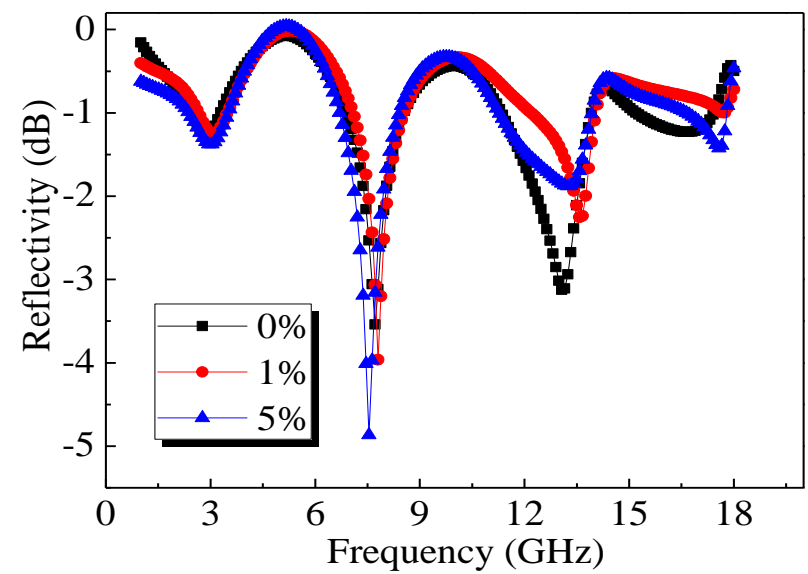

Fig. 10 Reflectivity of cementitious composites filled with NGPs 


\section{Table captions}

2 Table1. Properties of NGPs

3 Table 2 Mix proportions of cementitious composites filled with NGPs

4 
Table1. Properties of NGPs

\begin{tabular}{ccccc}
\hline Parameters & Diameter & Thickness & Specific surface area & Bulk density \\
\hline Value & $<2 \mu \mathrm{m}$ & $1-5 \mathrm{~nm}$ & $500 \mathrm{~m}^{2} / \mathrm{g}$ & $0-0.4 \mathrm{~g} / \mathrm{cm}^{3}$ \\
\hline
\end{tabular}

3

Table 2 Mix proportions of cementitious composites filled with NGPs

\begin{tabular}{ccccc}
\hline $\begin{array}{c}\text { Sample } \\
\text { number }\end{array}$ & $\begin{array}{c}\text { NGP content } \\
\text { (vol.\%) }\end{array}$ & $\begin{array}{c}\text { Water to cement } \\
\text { ratio }\end{array}$ & $\begin{array}{c}\text { Superplasticizer content } \\
\text { (vol.\%) }\end{array}$ & $\begin{array}{c}\text { Sand to cement } \\
\text { ratio }\end{array}$ \\
\hline NO.1 & 0 & 0.6 & 3 & 3 \\
NO.2 & 1 & 0.6 & 3 & 3 \\
NO.3 & 5 & 0.6 & 3 & 3 \\
\hline
\end{tabular}

4

5

6 